\title{
Pathogenesis study of enterovirus 71 infection in rhesus monkeys
}

\author{
Ying Zhang ${ }^{1}$, Wei Cui ${ }^{1}$, Longding Liu, Jingjing Wang, Hongling Zhao, Yun Liao, Ruixiong Na, \\ Chenghong Dong, Lichun Wang, Zhongping Xie, Jiahong Gao, Pingfang Cui, Xuemei Zhang and Qihan Li
}

Enterovirus 71 (EV71), a major pathogen that is responsible for causing hand-foot-and-mouth disease (HFMD) worldwide, is a member of the Human Enterovirus species A, family Picornaviridae. HFMD that is caused by EV71 is usually characterized by vesicular lesions on the skin and oral mucosa and high morbidity rates in children; additionally, occasional fatal cases have been reported involving brainstem encephalitis and myelitis associated with cardiopulmonary collapse. Although viral pathogenesis in humans is unclear, previous animal studies have indicated that EV71, inoculated via various routes, is capable of targeting and injuring the central nervous system (CNS). We report here the pathogenic process of systemic EV71 infection in rhesus monkeys after inoculation via intracerebral, intravenous, respiratory and digestive routes. Infection with EV71 via these routes resulted in different rates of targeting to and injury of the CNS. Intracerebral inoculation resulted in pulmonary edema and hemorrhage, along with impairment of neurons. However, intravenous and respiratory inoculations resulted in a direct infection of the CNS, accompanied by obvious inflammation of lung tissue, as shown by impairment of the alveoli structure and massive cellular infiltration around the terminal bronchioles and small vessels. These pathological changes were associated with a peak of viremia and dynamic viral distribution in organs over time in the infected monkeys. Our results suggest that the rhesus monkey model may be used to study not only the basic pathogenesis of EV71 viral infections, but also to examine clinical features, such as neurological lesions, in the CNS and pathological changes in associated organs.

Laboratory Investigation (2011) 91, 1337-1350; doi:10.1038/labinvest.2011.82; published online 9 May 2011

KEYWORDS: central nervous system (CNS); Enterovirus 71 (EV71); pathogenesis; pathology; rhesus monkeys

Enterovirus 71 (EV71) was first identified in 1969 (see Huang et $a l^{1}$ ) and is classified, along with some Coxsackie A viruses, as a member of the Human Enterovirus species A, family Picornaviridae. $^{2}$ This virus is the major pathogen that is responsible for the epidemics of hand-foot-and-mouth disease (HFMD). ${ }^{3}$ HFMD caused by EV71 is usually characterized by vesicular lesions on the skin and oral mucosa and high morbidity rates in children; ${ }^{4}$ its clinical presentation is similar to that of infection with the Coxsackie A16 (CA16) and Coxsackie 10 (CA10) viruses. ${ }^{5}$ Since the late 1990s, a series of large HFMD epidemics caused by EV71 have been reported in the Asia-Pacific region. ${ }^{6-8}$ EV71 genotypes B and $\mathrm{C}$, as well as $>10$ sub-genotypes, were isolated during these epidemics. ${ }^{9,10}$ Occasionally, EV71 infection clinically presents with neurological manifestations, in addition to other severe complications. ${ }^{11}$ Previous reports have shown that there were greater numbers of fatal cases of HFMD involving brainstem encephalitis, pulmonary edema and/or hemorrhage, and cardiopulmonary collapse in epidemics in Taiwan and Western Australia from 1997 to 2002. ${ }^{11,12}$ Patients who died during a severe EV71 epidemic of EV71 infection in Fuyang, China, in 2008 showed similar clinical manifestations of neural injury and pulmonary collapse. ${ }^{13,14}$

Epidemiological and clinical analyses of EV71 infections recorded over the past three decades have revealed that EV71 infection has a complex pathogenesis and that the central nervous system (CNS) is likely the main target of the EV71 virus. ${ }^{15}$ The mechanism that underlies this viral infection must be further investigated to develop clinical measures and specific vaccines against EV71. Although the available human clinical and pathological data have not explained this mechanism, the following observations might provide some

Institute of Medical Biology, Chinese Academy of Medical Science and Peking Union Medical College, Kunming, People's Republic of China

Correspondence: Professor Q Li, MD, PhD, Institute of Medical Biology, Chinese Academy of Medicine Science and Peking Union Medical College, 935 Jiaoling Road, Kunming, Yunnan, 650118, People's Republic of China.

E-mail: imbcams.lq@gmail.com

${ }^{1}$ These authors contributed equally to this work.

Received 22 November 2010; revised 25 March 2011; accepted 30 March 2011 
clues: first, EV71 infection can be transmitted via different routes within a population; ${ }^{16}$ second, the virus has been isolated from bloody secretions from the respiratory tract of an individual with clinical symptoms; ${ }^{17}$ and third, an infection of the CNS by EV71 suggests that circulation in the blood might be one of the ways the virus disseminates within the body. These findings suggest that the virus initially proliferates in a specific organ or tissue, with viremia occurring after this process, and, finally, damage of target organs by viral infection occurs. This inference is supported by work performed in animal models, such as cynomolgus monkeys and mice, in which intravenous or oral EV71 inoculation led to pathological changes in the CNS, including neuronal damage, in addition to the typical symptoms. ${ }^{18,19}$ Although these studies did not reveal any specific cardiopulmonary collapse or pulmonary edema in response to CNS failure, the fact that EV71 targeted the CNS through circulation and other ways provides a basis for further research.

To investigate the detailed pathogenesis of EV71 infection, we studied the pathological changes of different organs, primarily the CNS and respiratory, circulatory and digestive organs, of rhesus monkeys that were infected intracerebrally, intravenously, orally or intratracheally. Our results demonstrate that infection with EV71 via these routes caused the virus to target and injure the CNS at different rates. The infection caused by intracerebral inoculation induced pulmonary edema and hemorrhage, along with impairment of neurons. However, intravenous inoculation resulted in direct infection of the CNS, accompanied by obvious inflammation of lung tissue, as shown by impairment of the alveoli structure and massive cellular infiltration around the terminal bronchioles and small vessels. Interestingly, the same pathological changes in lungs were observed in monkeys infected with EV71 via the respiratory and digestive tracts, but infection via these routes resulted in low levels of pathological damage to the CNS. These results suggest that EV71 infection, at least in rhesus monkeys, progresses through the respiratory and digestive tracts, and the respiratory tract is likely the natural route of this infection. The data presented here provide a hypothesis for the EV71 infection process in rhesus monkeys and facilitates an understanding of EV71 transmission during epidemics as well as the clinical process of EV71 infection.

\section{MATERIALS AND METHODS}

\section{Cells and Viruses}

Monolayers of Vero cells were grown in Dulbecco's minimal essential medium (DMEM) supplemented with $5 \%$ calf serum, maintained at $37^{\circ} \mathrm{C}$ in flasks, and used for EV71 virus production and virus titration after inoculation in 96-well plates. The EV71 FY-23 strain was isolated from a hematic secretion of the respiratory tract of an infected male child who presented with the clinical symptoms of severe cardiopulmonary collapse during an epidemic in Fuyang, China, in May, 2008. ${ }^{13}$ The strain was sterilized using a $0.2-\mu \mathrm{m}$ filter and identified as an EV71 C4 sub-genotype virus by RT-PCR before we used the virus to inoculate Vero cells. The infected Vero cells were harvested and passaged continuously, and the third-passage cultures were further confirmed by a neutralization test using an antibody against the EV71 $\mathrm{BrCr}$ strain. This virus was used for all of the experiments.

\section{Rhesus Monkeys}

The experimental animal procedure was approved by the Office of Laboratory Animal Management of Yunnan Province, China. A total of 32 healthy monkeys with an average weight of $4.0 \pm 0.5 \mathrm{~kg}$ and an average age of 3-3.5 years were divided into four experimental groups; eight additional monkeys were used as blank controls, and three additional monkeys were used as negative controls. Each animal was kept in a single cage and bred according to the guidelines of the committee of experimental animals at the Institute of Medical Biology, Chinese Academy of Medical Sciences (CAMS). ${ }^{20}$ All animals were kept in isolation for 2 weeks before the initiation of the study. A neutralization test was conducted to confirm that they did not have antibodies against EV71 before the experimental infections.

\section{Infection of the Rhesus Monkeys with EV71}

Four experimental groups, each comprising eight monkeys, were set up. In each group, four monkeys were inoculated with $10^{6.5} \mathrm{CCID}_{50}$ (cell culture infectious doses) and four were inoculated with $10^{4.5} \mathrm{CCID}_{50}$ via intracerebral, intravenous injection or aerosol administration by fiberoptic bronchoscopy in the respiratory tract (detailed protocol described below). The same two doses were administered orally in water to eight additional monkeys. Intracerebral inoculation was performed according to the standard protocol for neurovirulence detection. ${ }^{21}$ In addition, three monkeys were used as negative controls. After viral inoculation, clinical manifestations were monitored and blood samples were collected daily. At 4, 7, 10 and 14 days post infection, two monkeys that were infected with each dose from each group were killed by electric shock, and the anatomical variations in these monkeys were examined. Organs from the cardiorespiratory system, digestive system and nervous system were collected for viral load detection. Organs were washed with phosphate-buffered saline (PBS) before homogenization.

\section{Infection with EV71 Through Fiberoptic Bronchoscopy}

Monkeys were anesthetized with ketamine $(10 \mathrm{mg} / \mathrm{kg}$ of body weight, Phoenix Pharmaceuticals, St Joseph, MO, USA) and infected by wedging the bronchoscope into the desired trachea. Viruses, which were contained in $2 \mathrm{ml}$ of sterile saline, were instilled via the biopsy channel of the bronchoscope.

\section{Viral Titration and Neutralization Test}

The viral titration of EV71 that was harvested from cell culture or isolated from various tissue homogenates (from the cardiorespiratory system, digestive system and nerve 
system) of infected animals was analyzed by microtitration assay $^{22}$ or PFU assay. Briefly, viral stocks were serially diluted 10 times, and the diluted stocks were added to six-well plates coated with Vero cells. Subsequently, pre-warmed $1 \times$ DMEM with $1 \%$ agar was added to the cell surface, and plates were incubated at $37^{\circ} \mathrm{C}$ for 4 to 5 days. Before the calculation of plaque formation, $4 \%$ paraformaldehyde and $0.5 \%$ crystal violet were added to each well for fixing and dyeing, respectively.

Neutralization of the viruses was performed according to the standard protocol. ${ }^{23}$ Briefly, a mixture of diluted serum containing anti-EV71 antibodies and virus with a titer of $500-1000 \mathrm{CCID}_{50}$ in $100 \mu \mathrm{l}$ of PBS were incubated at $37^{\circ} \mathrm{C}$ for $1 \mathrm{~h}$, and the cellular pathogenic effect (CPE) of the viruses was examined by inoculating this mixture into Vero cells grown in 96-well plates.

\section{Histopathological and Immunohistochemical Analyses}

Various tissue samples from the organs of the infected monkeys, including the brain, spinal cord, heart, lungs, liver, spleen, kidneys and lymphatic system, were fixed in 10\% formalin in PBS, dehydrated in ethanol gradients, and embedded in paraffin before obtaining $4-\mu \mathrm{m}$ sections for further hematoxylin and eosin (H\&E) staining. CNS tissue samples were also treated by Kluver-Barrera Luxol fast blue (LFB) staining. ${ }^{24}$ Histopathological analysis of the tissue sections from each organ was performed under a light microscope. Histological changes in the CNS (lesion score) were evaluated using a method recommended by the World Health Organization (WHO), ${ }^{25}$ which included standard pathological evaluations of the cerebrum, cerebellum, brain stem and different sections of the spinal cord. ${ }^{18}$

For immunohistochemical analysis, tissue samples were embedded in an optimal cutting temperature (OCT) compound (Miles, Elkhart, IN, USA) and frozen in liquid nitrogen. The frozen tissue samples were then cut into $4-\mu \mathrm{m}$ sections, placed on poly-L-lysine-coated glass slides and fixed in 3.7\% paraformaldehyde. The endogenous peroxidase activity of the tissues was inhibited by treatment with hydrogen peroxide $(2.5 \%)$. EV71 antigen was detected by mouse antiEV71 monoclonal antibody (Clone 10F0; Abcam, Cambridge, MA, USA) and horseradish peroxidase (HRP)-conjugated anti-mouse IgG antibodies (Sigma, Deisenhofen, Germany), followed by color development with diaminobenzidine for the detection of the antigen-antibody reaction.

\section{Viral RNA Extraction and Real-Time RT-PCR Assay}

Viral RNA was extracted from $250 \mu \mathrm{l}$ of fresh tissue homogenate $(10 \%)$ and fecal homogenate $(5 \%)$ from experimental animals using the Qiagen Rneasy mini kit, according to the manufacturer's protocol (Qiagen, Hilden, Germany). Real-time RT-PCR assays were performed using the One Step PrimeScript RT-PCR Kit (Takara Shuzo, Shiga, Japan) in the 7500 Fast Real-time RT-PCR system (Applied Biosystems, Foster City, CA, USA). The reaction mixture contained the
Taqman Universal PCR master mix, each primer at $200 \mathrm{nM}$, the FAM/TAMRA-labeled probe at $100 \mathrm{nM}$ (Takara Shuzo) and $2 \mu \mathrm{l}$ of RNA in a $20-\mu \mathrm{l}$ total reaction volume. The sequences for the EV71-specific primers were as follows: forward primer, 5'-CCCTGAATGCGGCTAATCC-3'; probe, $5^{\prime}$-CCAGCGGGTAGTGTGTCGTAACGGG- $3^{\prime}$; and reverse primer, $5^{\prime}$-ATTGTCACCATAAGCAGCCA- ${ }^{\prime}$. The following protocol was used for all PCR assays: $5 \mathrm{~min}$ at $42^{\circ} \mathrm{C}$ and $10 \mathrm{~s}$ at $95^{\circ} \mathrm{C}$, followed by 40 cycles of $95^{\circ} \mathrm{C}$ for $5 \mathrm{~s}$ and $60^{\circ} \mathrm{C}$ for $30 \mathrm{~s}$. A standard reference curve was obtained by measurement of the serially diluted virus RNA that was generated by in vitro transcription from a DNA construct that contained the $5^{\prime}$-untranslated region (UTR). Based on the copy number of standard RNA, we then quantified the viral titer relative to this standard RNA by real-time RT-PCR analysis. Using this approach, the viral load was measured separately in each infected tissue.

\section{Statistical Analysis}

Data obtained from all experiments were analyzed by $t$-test for three samples, and a $P$-value of $<0.05$ was considered significant. Data are expressed as the average of two samples in some experiments.

\section{RESULTS \\ Clinical Manifestations of EV71 Infection in Rhesus Monkeys Infected via Four Different Routes}

It was previously reported that EV71 infection can induce neurological lesions in the CNS of cynomolgus monkeys and that these lesions manifest clinically as significantly lower muscle tension in the limbs, flaccid paralysis and ataxia. ${ }^{6,26,27}$ These symptoms suggest the presence of lesions in the CNS of the monkeys. In this study, physical examination of the monkeys that were infected via these four routes revealed that the muscle tension in the limbs was in the normal range, and no herpes-like skin lesions were observed on the hands, feet or mouths. Nevertheless, twice-daily measurement of body temperature revealed that monkeys that were infected through the intracerebral, intravenous and respiratory routes had body temperatures $1-1.5^{\circ} \mathrm{C}$ higher than normal (Figure 1a). This increase in body temperature lasted from days 4 to 5 post infection until days 7 to 8 post infection, at which time the monkeys recovered. There was no distinct body temperature difference between the groups that received the two different doses. This increase in body temperature was not observed in the monkeys that were infected orally (Figure 1a). Blood tests, performed daily, indicated that mononuclear cells in the peripheral blood were highly elevated from day 3 post infection until the end of the experiment (Figure $1 \mathrm{~b}$ ) in all of the infected monkeys except those infected via the digestive tract (Figure 1b). Levels of inflammatory cytokines, including interleukin-2 (IL-2), tumor necrosis factor- $\alpha$ (TNF- $\alpha$ ), interferon- $\gamma$ (IFN- $\gamma$ ) and interleukin-6 (IL-6), in monkeys that were infected via all 

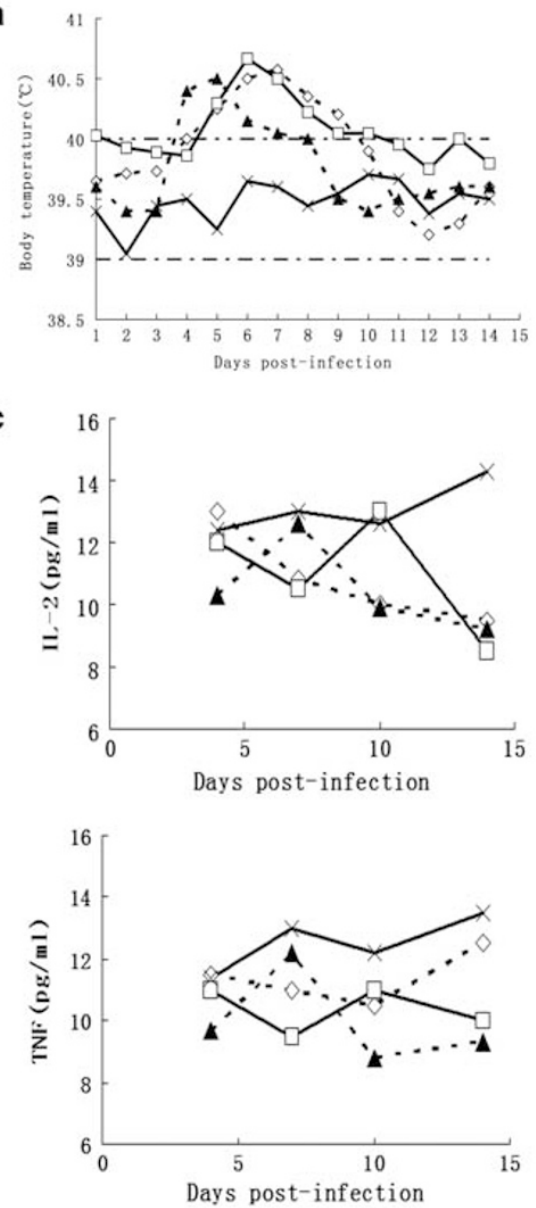

b
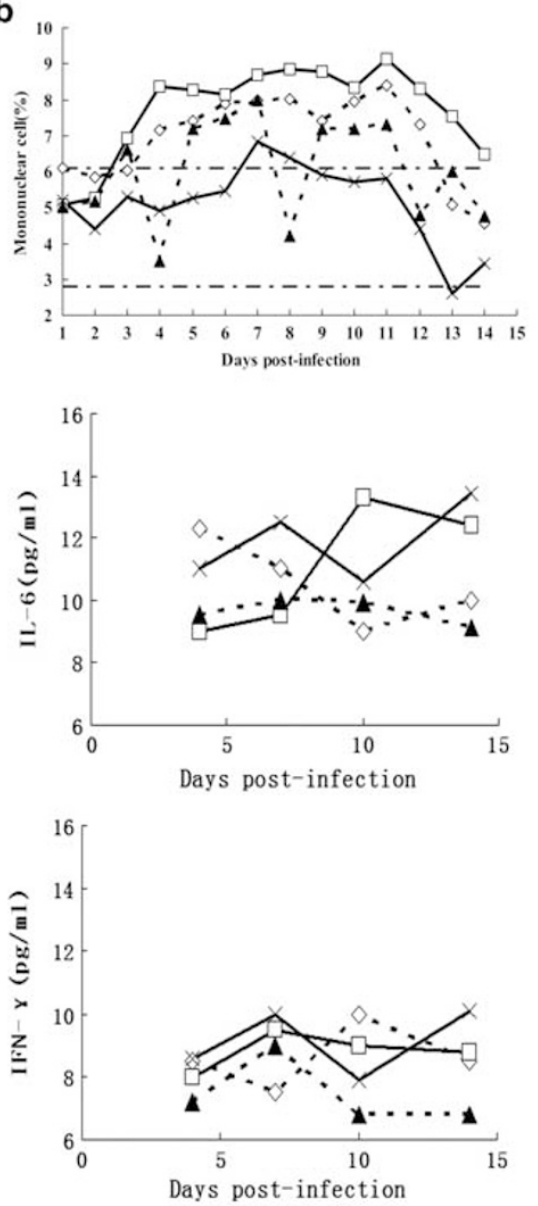

$\bullet \bullet$ Intravenous infection $\quad-\square-$ Intracerebral infection

- Respiratory infection $\longrightarrow$ Digestive infection

Figure 1 Clinical manifestations in EV71 intracerebral-, intravenous-, respiratory- and digestive route-infected rhesus monkeys. (a) Monitoring of body temperatures of rhesus monkeys infected by EV71 through four routes. Body temperatures of infected monkeys were measured by anus route twice each day post infection. The normal body temperatures ranging from 39 to $40^{\circ} \mathrm{C}$ are shown as dot line. (b) FCM and number statistic analysis of mononuclear cells in blood specimens of rhesus monkeys infected by EV71. The percentages of normal mononuclear cells ranging from 2.9 to 6.1 are shown as dot line. (c) FCM analysis of the inflammatory markers, that is, IL-2, IL-6, TNF, $\gamma$ IFN and so on cytokines of rhesus monkeys infected by EV71. Blood samples were collected on 4, 7, 10 and 14 days post infection for detecting the presence of certain inflammatory markers. Values are the mean for two samples at each time point.

four routes exhibited slight variations that were within the normal ranges (Figure 1c).

\section{Kinetics of Viremia in EV71-Infected Rhesus Monkeys That Were Infected via the Four Routes}

No definitive data have been published on the kinetics of viremia in EV71-infected humans because of difficulties in collecting blood specimens throughout the course of the infection. Our study, based on blood samples taken from monkeys that were infected via four different routes, revealed several interesting facts. Viral loads with relatively high peaks of $\left(\sim 10^{3}\right.$ copies $\left./ \mathrm{ml}\right)$ were detected in monkeys that were infected via the intravenous and respiratory routes from day 4 to day 7 post infection (Figure $2 a$ ). The distribution of the viral load in the blood of monkeys that were infected via the respiratory and intravenous routes displayed a typical viremia (Figure 2a). Oral inoculation induced a slight viremia with relatively low viral loads $\left(<10^{2}\right.$ copies/ml) between days 4 and 7 post infection (Figure 2a). Only a transient viremia, with a very low viral load $\left(10^{1}\right.$ copies $\left./ \mathrm{ml}\right)$, was observed within a reliable range on day 4 post infection in monkeys that were infected via the intracerebral route (Figure $2 a$ ). To confirm this detection, virus from the same sample was titered on Vero cells. Indeed, results from the titer experiment confirmed the viral load data (Figure 2b). Higher viral titers were consistently observed in monkeys that were infected via the intravenous and respiratory routes from days 4 to 7 post infection (Figure 2b).

\section{Viral Load Variations in the Organs of EV71-Infected Rhesus Monkeys}

To understand viral transmission and distribution in EV71infected monkeys, the viral loads in various organs were measured in monkeys that were killed on days 4, 7, 10 and 14 

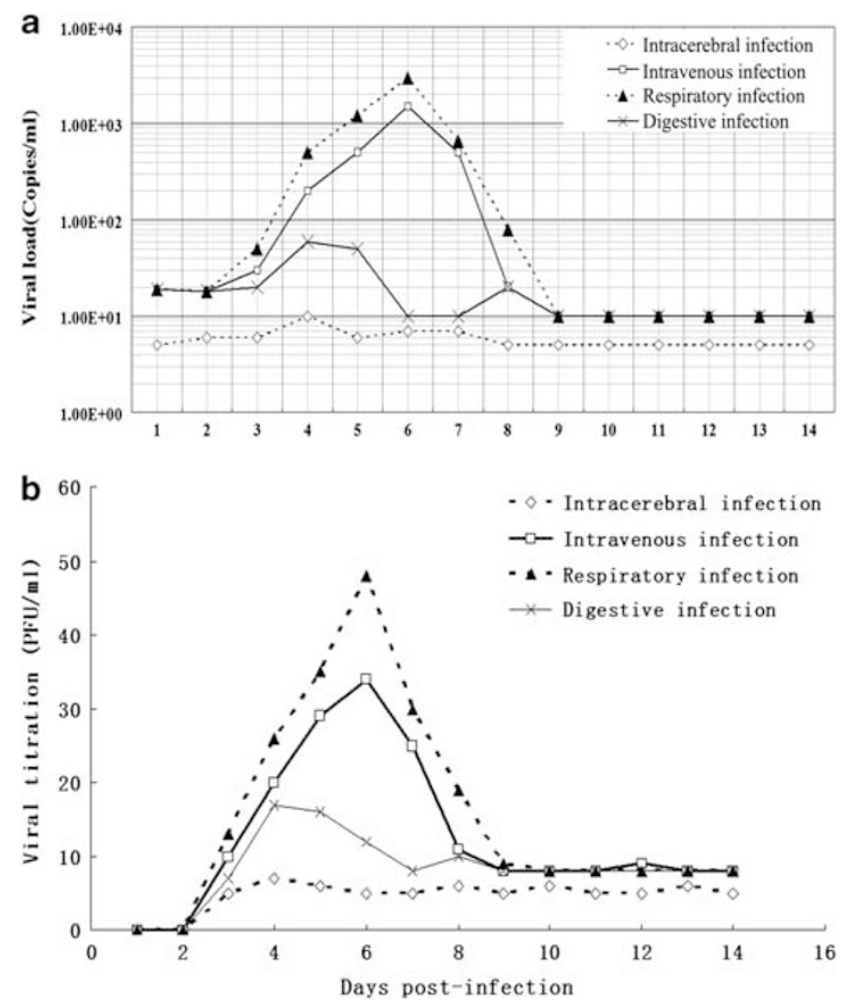

Figure 2 Viral load variations in rhesus monkeys infected via intracerebral, intravenous, respiratory and digestive routes. Blood specimens were collected in whole course of EV71 infection (1-14 days post infection). (a) Viral RNA was extracted from blood specimens and measured by Taqman-based real-time qPCR assay, according to the procedure as described in the manufacture's protocol. Viral copies were quantified according to vitro-synthesized RNA by spectrophotometry quantification, and the quantity was expressed as a relative copy number, determined by the equation $(\mu \mathrm{g}$ of $\mathrm{RNA} / \mu \mathrm{l}) /($ molecular weight $)) \times$ Avogadro's number $=$ viral copy number/ $\mu \mathrm{l}$. (b) Viral titration was extracted from blood specimens and measured by a plague assay, according to the procedure as described in the Materials and methods. Values are the mean for two samples at each time point.

post infection. Starting at day 4 post infection, we observed a relatively high viral load of $\sim 10^{4-5}$ copies per $100 \mathrm{mg}$ in some lymph nodes, such as the axillary lymph nodes, of the monkeys that were infected via the respiratory, intracerebral and intravenous routes; this viral load was maintained until days 7 to 10 post infection (Figure 3a). Approximately $10^{3-4}$ copies per $100 \mathrm{mg}$ were detected in other lymph nodes, such as the lower neck and inguinal lymph nodes (Figure 3a), whereas only $10^{1-2}$ copies per $100 \mathrm{mg}$ were detected in lung lymph nodes and in the mesenteric lymph nodes between days 7 and 10 post infection. Relatively high viral loads were observed in the spleen between days 4 and 7 post infection (Figure 3a). Similarly, in monkeys that were infected orally, the highest viral loads were also detected in the axillary lymph nodes $\left(10^{2}\right.$ copies per $\left.100 \mathrm{mg}\right)$, and relatively low viral loads were detected in the lower neck, spleen and mesenteric lymph nodes $\left(<10^{2}\right.$ copies per $100 \mathrm{mg}$; Figure 3a). Interestingly, there was no virus detected in the spleen or most lymph nodes, except for the axillary lymph node, of monkeys that were infected via the intracerebral route (Figure 3a).

An analysis of the CNS showed that viral loads increased, to some extent, from day 4 post infection in monkeys that were infected via all routes except orally. In the intracerebral-, intravenous- and respiratory route-infected groups, $\sim 10^{3}$ copies per $100 \mathrm{mg}$ were detected in the thalamus and cerebellum, and $\sim 10^{4}$ copies per $100 \mathrm{mg}$ were detected in the spinal cord (Figure $3 \mathrm{~b}$ ). Slightly higher levels of $10^{1-2}$ copies per $100 \mathrm{mg}$ were observed in the cerebral and spinal cord tissue of the group that was infected via the digestive route (Figure 3b). Notably, viral loads in the four groups reached maximum values on day 7 post infection, after which time they gradually decreased (Figure $3 \mathrm{~b}$ ). The results from the intracerebral, intravenous and respiratory inoculation groups indicated that viral loads in the CNS corresponded well with the establishment of viremia.

Interestingly, virus was detected in some glands and major organs of the monkeys that were infected via the intravenous, respiratory and intestinal routes (Figure $3 \mathrm{c}$ ) but not in monkeys infected via the intracerebral route (Figure 3c). High viral loads were observed in some organs, such as the bronchial tubes and pancreas (Figure 3c), whereas low viral loads were detected in the stomach and other intestinal organs of all monkeys except those that were infected via intracerebral inoculation (Figure 3c).

It is particularly important to note that the peak viral loads in all organs were observed at approximately day 7 post infection, after which time the established viremia began to decrease until it gradually disappeared.

\section{Neurological Lesions in EV71-Infected Rhesus Monkeys}

Previous histopathological studies of tissue specimens collected from fatal human cases of EV71 infection and from murine models of infection have demonstrated that the neuropathological changes resulting from neuron lesions and regional inflammatory responses were the cause of death. ${ }^{15,26}$ Neuropathological investigations during autopsy and data from murine models have indicated that inflammation was the most marked in spinal cord gray matter, the brainstem and the hypothalamus. ${ }^{28,29}$ In this study, histopathological examination of the monkeys provided kinetic data for neuropathological analysis. In monkeys that were infected via the intracerebral route, virus-induced pathological changes were observed mainly in the subthalamus, the brainstem and the hypothalamus. These changes included the following: obvious infiltration of inflammatory cells in the caudate nucleus, pontine nucleus and substantia nigra; neurons retrogression; and pronounced perivascular cuffing (Figure 4a). During the 14-day experiment, the lesions in these parts of the brain gradually worsened, until they reached the peak of their severity at day 10 post infection (Table 1). In other parts of the cerebrum, similar pathological changes of various levels of severity were found (Figure $4 \mathrm{~b}$ ). The pathological changes that were observed in the spinal cord tissues of all of 


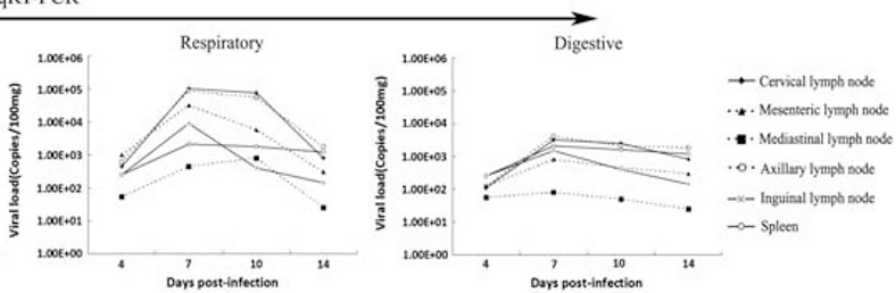

b

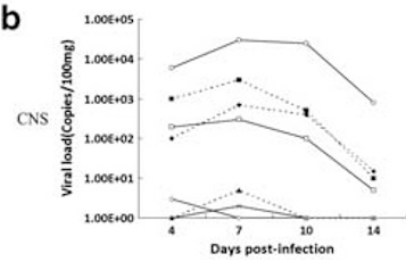

C

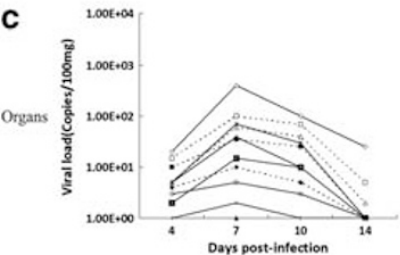

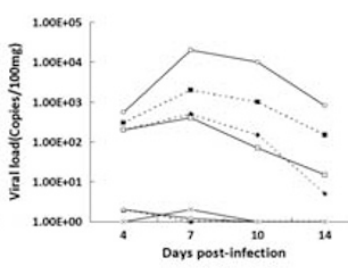
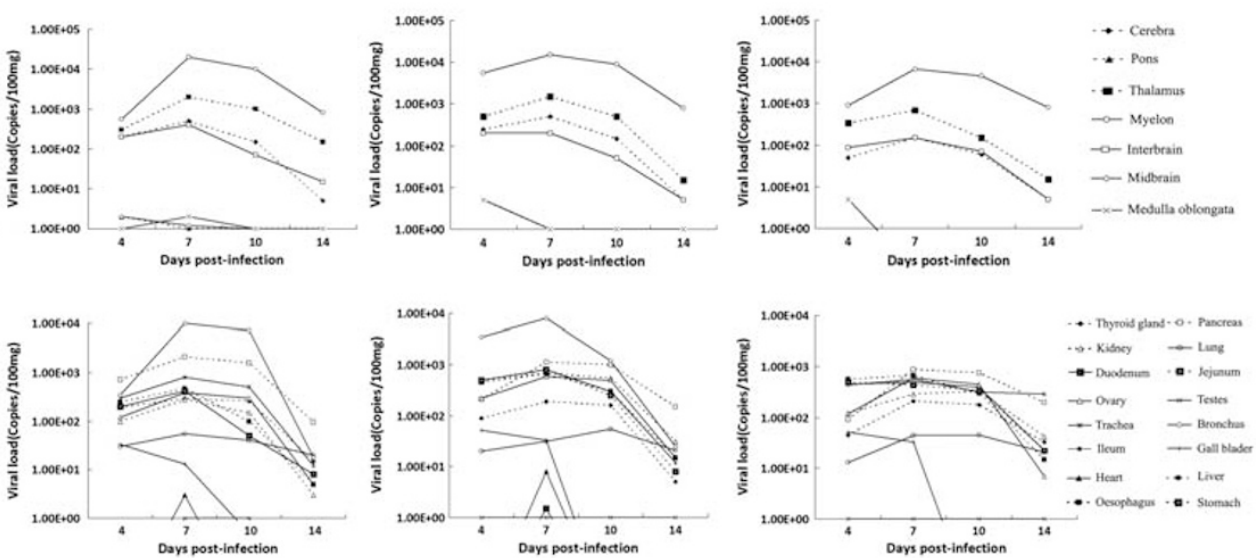

d
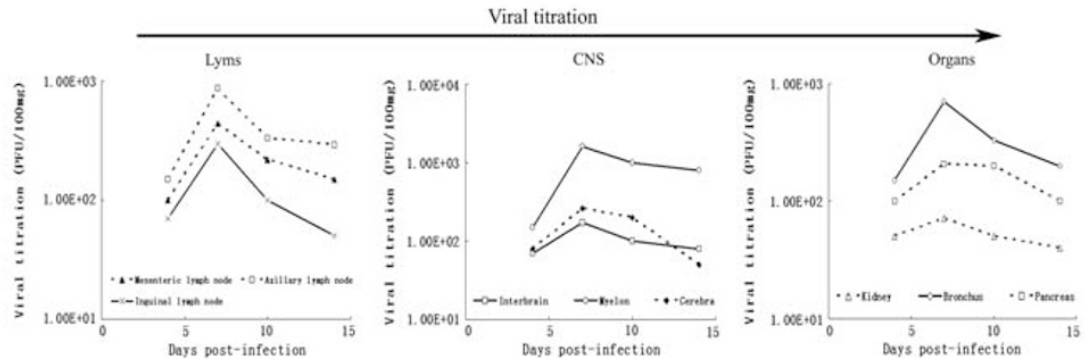

Figure 3 Comparison of viral load in different organs of rhesus monkeys infected via intracerebral, intravenous, respiratory and digestive routes, which were measured by Taqman-based real-time qPCR assay and viral titration in Vero cell. Viral copies were quantified according to vitro-synthesized RNA by spectrophotometry quantification, and the quantity was expressed as a relative copy number, determined by the equation $(\mu \mathrm{g}$ of RNA/ $\mu \mathrm{l}) /(\mathrm{molecular}$ weight) $\times$ Avogadro's number $=$ viral copy number/ $\mu$ l. (a) Viral load in lymphatic system of infected rhesus monkeys (cervical lymph node, axillary lymph node, mesenteric lymph node, inguinal lymph node, mediastinal lymph node and spleen). (b) Viral load in CNS of infected rhesus monkeys (cerebra, interbrain, pons, midbrain, thalamus, medulla oblongata and myelon). (c) Viral load in the other major organs of endocrine system, respiratory apparatus and alimentary system of infected rhesus monkeys (thyroid gland, pancreas, ovary, testes, heart, kidney, lung, trachea, bronchus, esophagus, duodenum, jejunum, ileum and gall bladder). (d) Viral titration in lymphatic system (axillary lymph node, mesenteric lymph node and inguinal lymph node), CNS (cerebra, interbrain and myelon) and other major organs (kidney, bronchus and pancreas) of infected rhesus monkeys through respiratory route. Tissue specimens were collected from organs of these systems on days 4, 7, 10 and 14 post infection, and homogenated in $10 \%$ suspension. Viral titer was determined by plague assay as described in the Materials and methods. Values are the mean for two samples at each time point.

the monkeys were defined as mild-to-moderate myelitis, and these changes showed the following features: (1) each part of the spinal cord, including the cervical, thorax and lumber regions, had similar lesions; (2) the pathological changes were distributed bilaterally in the spinal cord; (3) histopathological examination revealed changes including the diffuse infiltration of inflammatory cells into the gray matter, the formation of glial nodules, perivascular cuffing and damaged neurons; and (4) the greatest changes in inflammation were observed in the anterior horn, and some of these changes were distributed bilaterally (Figure $4 \mathrm{c}$ and Supplementary Figure 1). Detailed examination of the 22 segments of the spinal cord revealed a comprehensive view of the neuropathological changes (Figure $4 \mathrm{~d}$ and Supplementary Figure 1). Lesions in the cerebrum progressed with kinetics similar to those that were observed for the spinal cord (Table 1).

In monkeys that were infected via the intravenous route, similar pathological changes were observed in the cerebrum, thalamus and spinal cord tissues of all monkeys, and these changes were defined as mild to moderate (Table 2). In monkeys that were infected via the respiratory route, the induced pathological changes were observed in the aforementioned tissues of $50 \%$ of the monkeys and were defined as slight to moderate cerebritis and/or myelitis (Table 2). In monkeys that were infected via the digestive route, mild 
virus-induced neurological lesions were observed in the cerebrum and midbrain of $25 \%$ of the monkeys, whereas slight myelitis occurred in another 25\% (Table 2).
These examinations also suggested that the virus-induced neurological lesions in the CNSs of the intracerebrally and intravenously infected monkeys were more severe than those

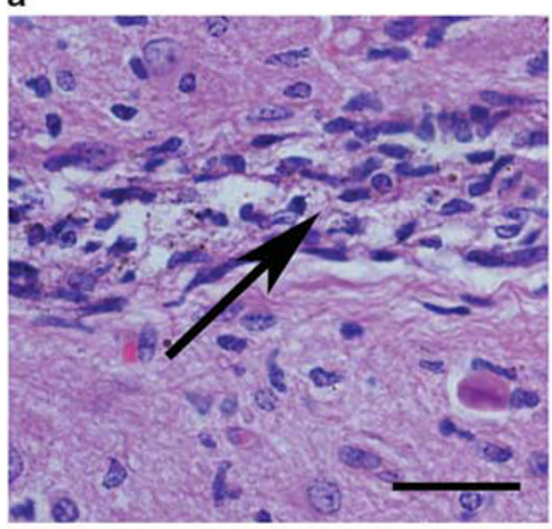

b

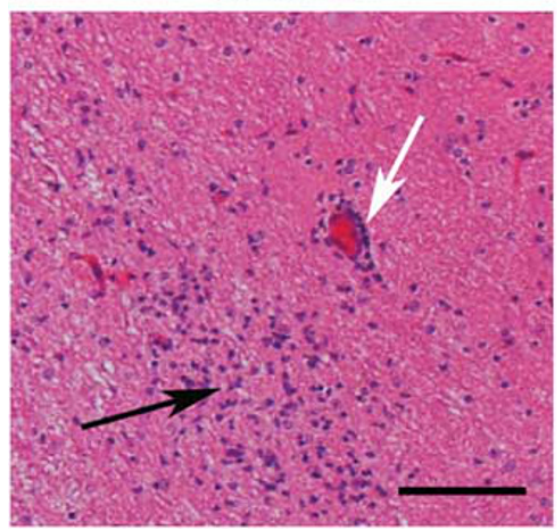

C

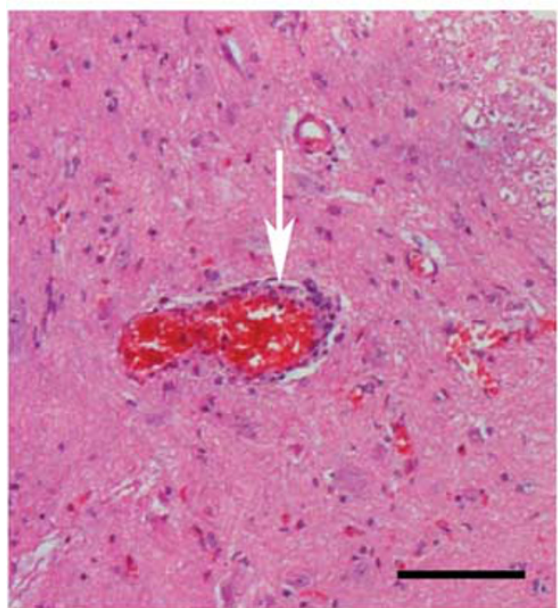

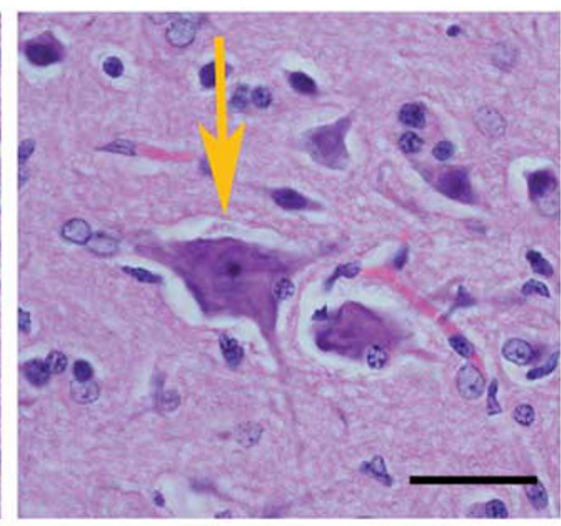

Pons
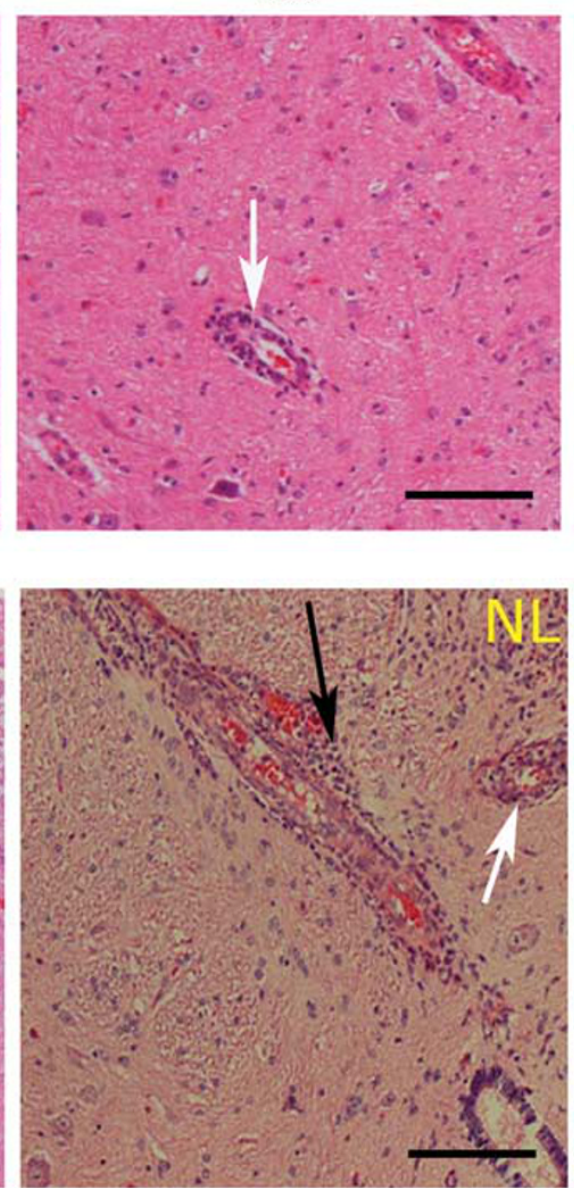

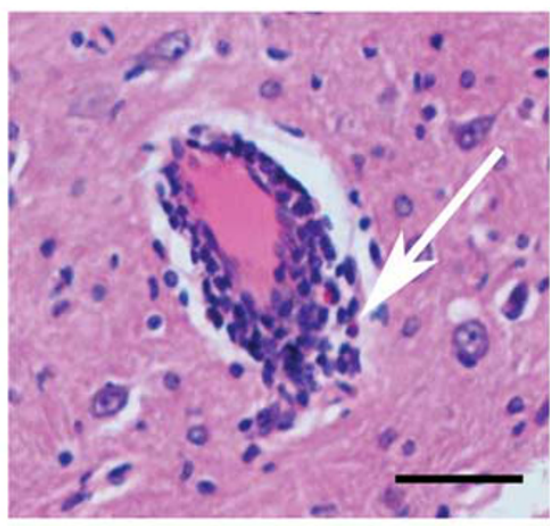

Oblongata

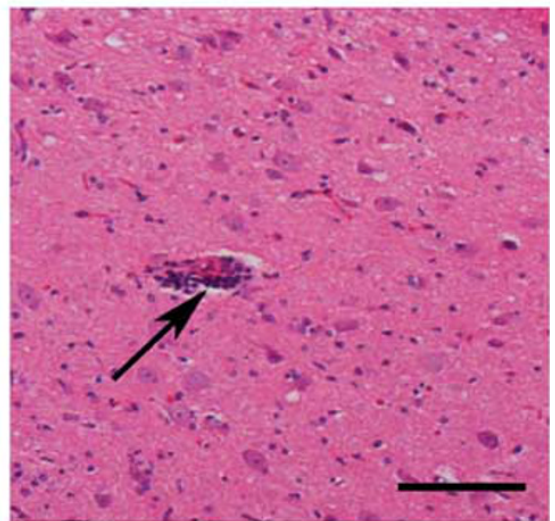

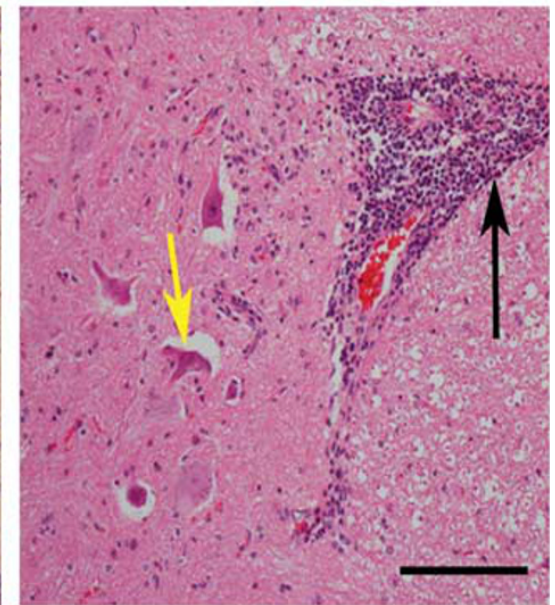

Figure 4 The neuropathological changes in EV71-infected rhesus monkeys through intracerebral, intravenous, respiratory and digestive routes. (a) The typical features of neuropathological changes in cerebra. Infiltration of inflammatory cells (black arrow), perivascular cuffing (white arrow) and neuron retrogression (yellow arrow). Magnification $\times 400$. Bar, $15 \mu \mathrm{m}$. (b) The features of neuropathological changes in other parts of cerebra (cerebellar, pons, medulla oblongata). Magnification $\times 100$. Bar, $60 \mu \mathrm{m}$. (c) The typical features of pathological changes in spinal cords. Infiltration of inflammatory cells (black arrow), neuron damaged (yellow arrow and neuronal lesion (NL) regions) and perivascular cuffing (white arrow). Magnification $\times 200$. Bar, $30 \mu \mathrm{m}$. (d) The features of neuropathological changes in spinal cords. Each part (cervical, thorax, lumbar) was showed by two segments. Magnification $\times 200$ (cervical and thorax (upper); Bar, $30 \mu \mathrm{m}$ ) and magnification $\times 100$ (thorax (lower) and lumbar; Bar, $60 \mu \mathrm{m}$ ). Samples of these tissues were collected on day 10 post infection. Histopathological examination of the tissue sections from each organ stained with hematoxylin and eosin (H\&E) was performed under a light microscope. 
d
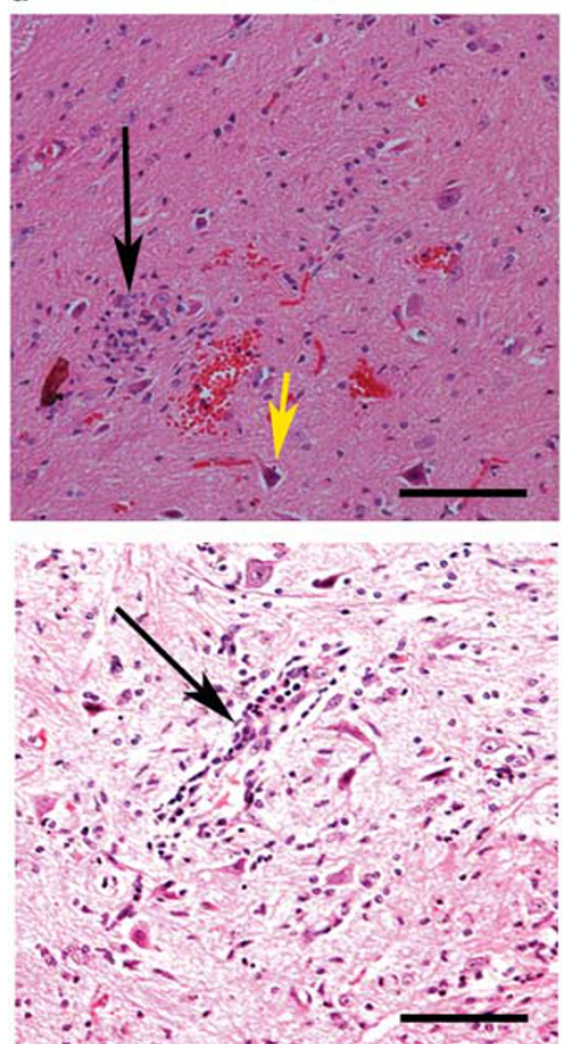

Thorax
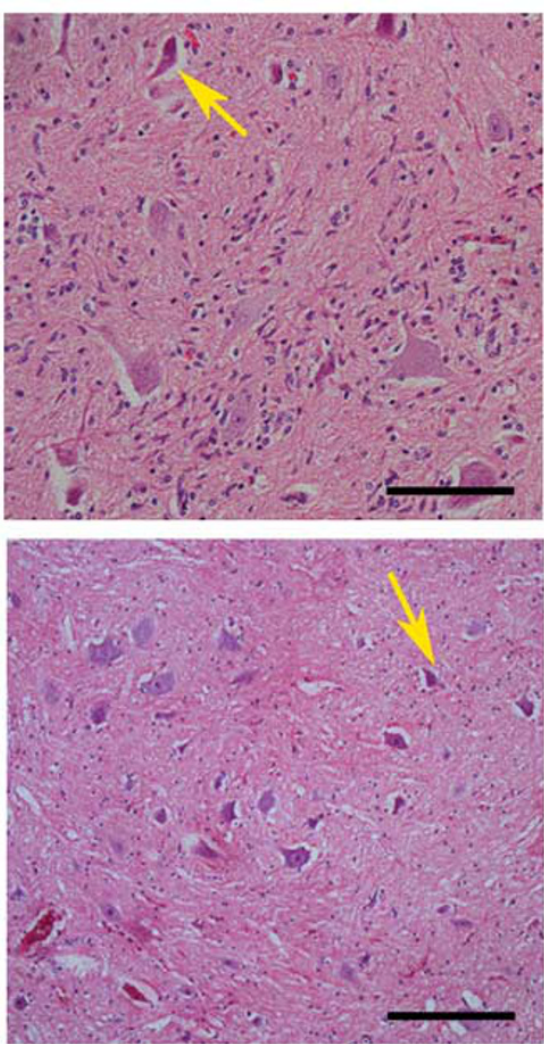

Lumbar
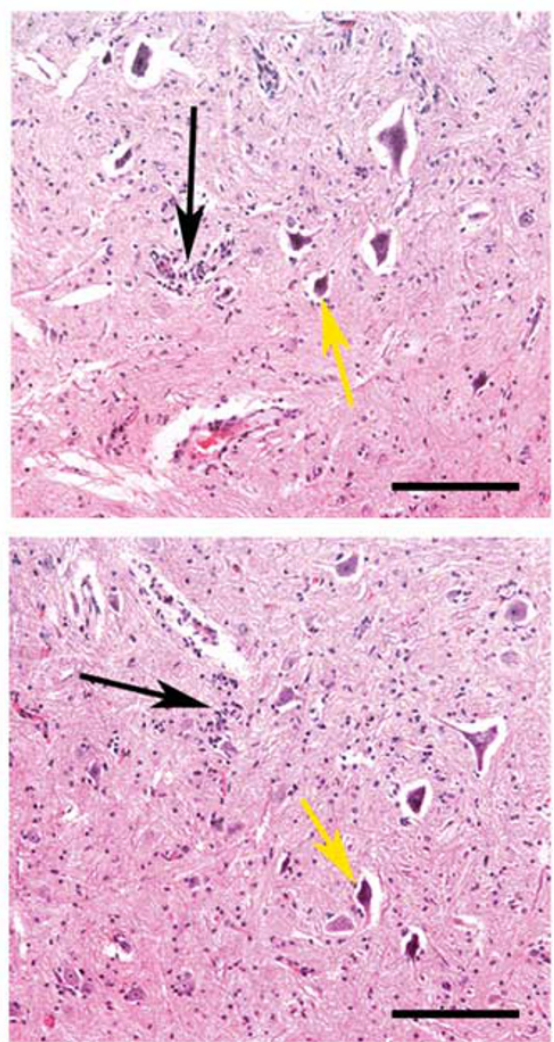

Figure 4 Continued

\section{Table 1 Neuropathological evaluation in Rhesus monkeys infected via intracerebral route}

Dose

Neuropathological changes ${ }^{a}$ (days post infection)

\begin{tabular}{cccc}
\hline Brain & Spinal cord \\
\hline Cerebra Interbrain Midbrain & $\begin{array}{c}\text { Medulla Cervical Thorax Lumbar } \\
\text { oblongata }\end{array}$ \\
\hline
\end{tabular}

$47101447101447101447 \quad 1014471014471014471014$ $\begin{array}{llllllllllllllllllllllllllll}10^{4.5} & 3 & 3 & 2 & 2 & 2 & 2 & 1 & 1 & 3 & 4 & 2 & 1 & 2 & 2 & 1 & 1 & 1 & 1 & 1 & 0 & 1 & 2 & 1 & 0 & 1 & 1 & 0\end{array}$

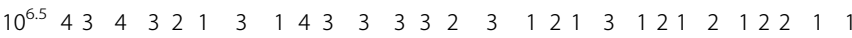

${ }^{\mathrm{a}} \mathrm{CNS}$ lesions were scored according to the evaluation of the extent of pathological change in CNS: 0 , no lesion; 1 , cellular infiltration; 2 , cellular infiltration with minimal neural damage; 3 , cellular infiltration with extensive neural damage; and 4, massive neural damage with or without cellular infiltration.

Each number represents the data from one animal.

of the monkeys that were infected via the respiratory route. The lesions in the monkeys that were infected via the digestive route were the least severe.

\section{Histopathological Examination of Major Organs of EV71-Infected Rhesus Monkeys}

Previous histopathological studies of clinical specimens that were collected from fatal human cases of EV71 infection re-
Table 2 Neuropathological evaluation in Rhesus monkeys infected via intravenous, respiratory or digestive routes

Infection route Dose $\frac{\text { Neuropathological } \text { changes }^{\mathrm{a}} \text { (days post infection) }}{$\cline { 3 - 4 } \text {$Cerebra Interbrain Cervical Thorax Lumbar }}$

$\begin{array}{llllllllllllllllllll}4 & 7 & 10 & 14 & 4 & 7 & 10 & 14 & 4 & 7 & 10 & 14 & 4 & 7 & 10 & 14 & 4 & 7 & 10 & 14\end{array}$

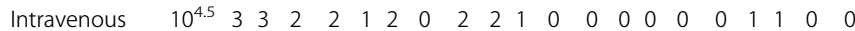
$\begin{array}{lllllllllllllllllllll}10^{6.5} & 4 & 2 & 3 & 3 & 2 & 1 & 2 & 1 & 2 & 0 & 2 & 0 & 1 & 1 & 1 & 0 & 1 & 0 & 0 & 0\end{array}$

$\begin{array}{llllllllllllllllllllll}\text { Respiratory } & 10^{4.5} & 1 & 4 & 0 & 0 & 1 & 1 & 0 & 0 & 1 & 2 & 1 & 0 & 1 & 2 & 0 & 1 & 1 & 2 & 2 & 1\end{array}$

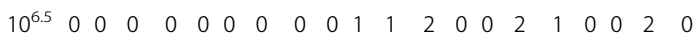

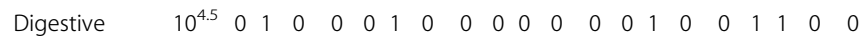

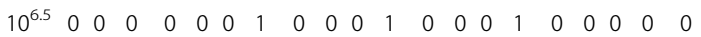

${ }^{\mathrm{a}} \mathrm{CNS}$ lesions were scored according to the evaluation of the extent of pathological change in CNS: 0 , no lesion; 1 , cellular infiltration; 2, cellular infiltration with minimal neural damage; 3 , cellular infiltration with extensive neural damage; and 4, massive neural damage with or without cellular infiltration.

Each number represents the data from one animal.

vealed that pulmonary edema, damage to lung tissue and inflammatory cell aggregation were present in the lung tissues of patients, ${ }^{26}$ and slight inflammatory lesions were found in 
cardiac tissue. ${ }^{30}$ In our histopathological examination of organs from monkeys that were infected with EV71, different pathological changes were present in the lung tissues of monkeys infected by each of the different routes.

In intracerebrally infected monkeys, lesions typical of pulmonary edema and slight hemorrhage around the hilum of the lungs were observed in $50 \%$ of the monkeys (Table 3 ). These histopathological changes in the lungs had the characteristics of hyperemia, as demonstrated by the extensive bronchial and alveolar exudates that were present in some parts of the sections marked with a deep pink color (Figure 5).

In contrast, the monkeys that were inoculated intravenously with either a high or a low dose of EV71 displayed a typical local inflammatory response and did not develop edema. The interstitial pneumonia that was observed in the lungs might lead to other pathological changes, such as alveolar damage, vessel hyperemia, massive cellular infiltration around the terminal bronchioles and proliferation of lymphocyte follicles (Table 3 and Figure 5).

Table 3 Pathological changes in Rhesus monkeys infected via intracerebral, intravenous, respiratory or digestive routes

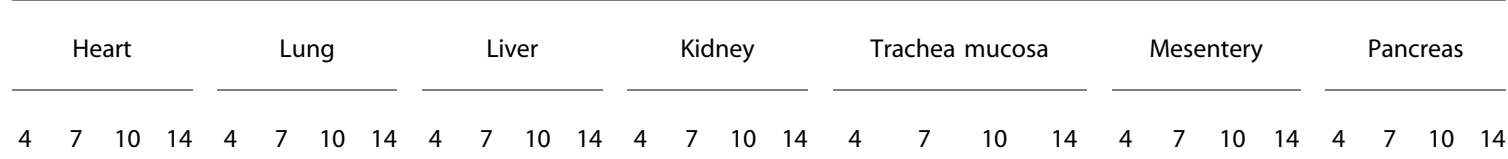

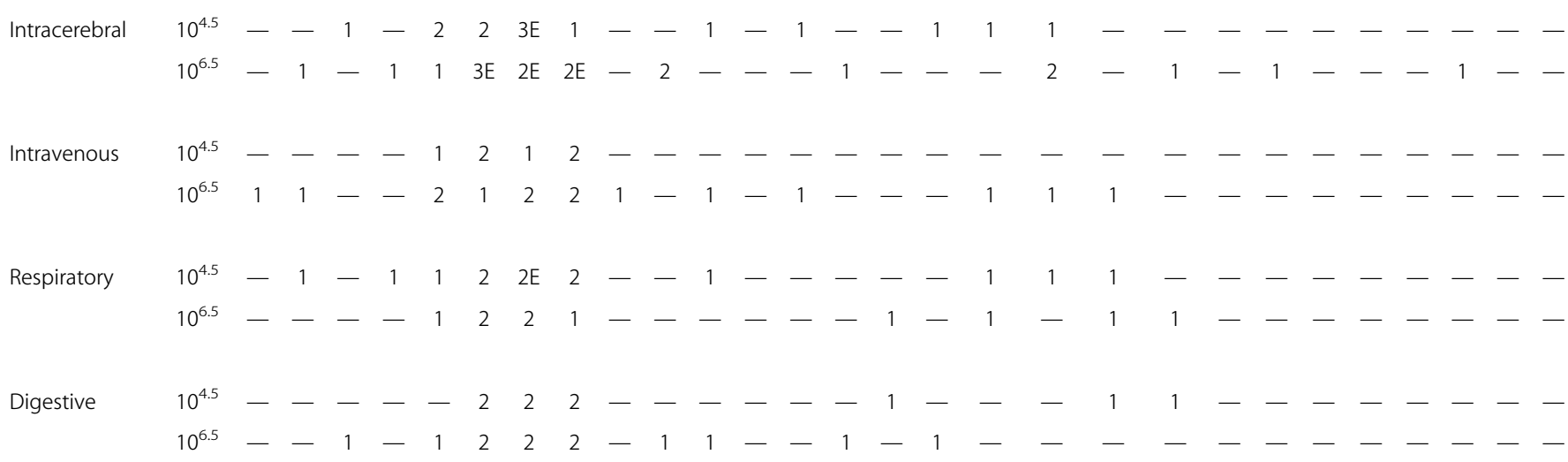

${ }^{a}$ E: edema and/or hemorrhage. 1: cellular infiltration; 2: cellular infiltration and damage of tissue; 3: massive cellular infiltration and severe damage of tissue; -: no obvious pathological change.

The data that are not reflected in the table have no significantly pathological change.

Each number represents the data from one animal.

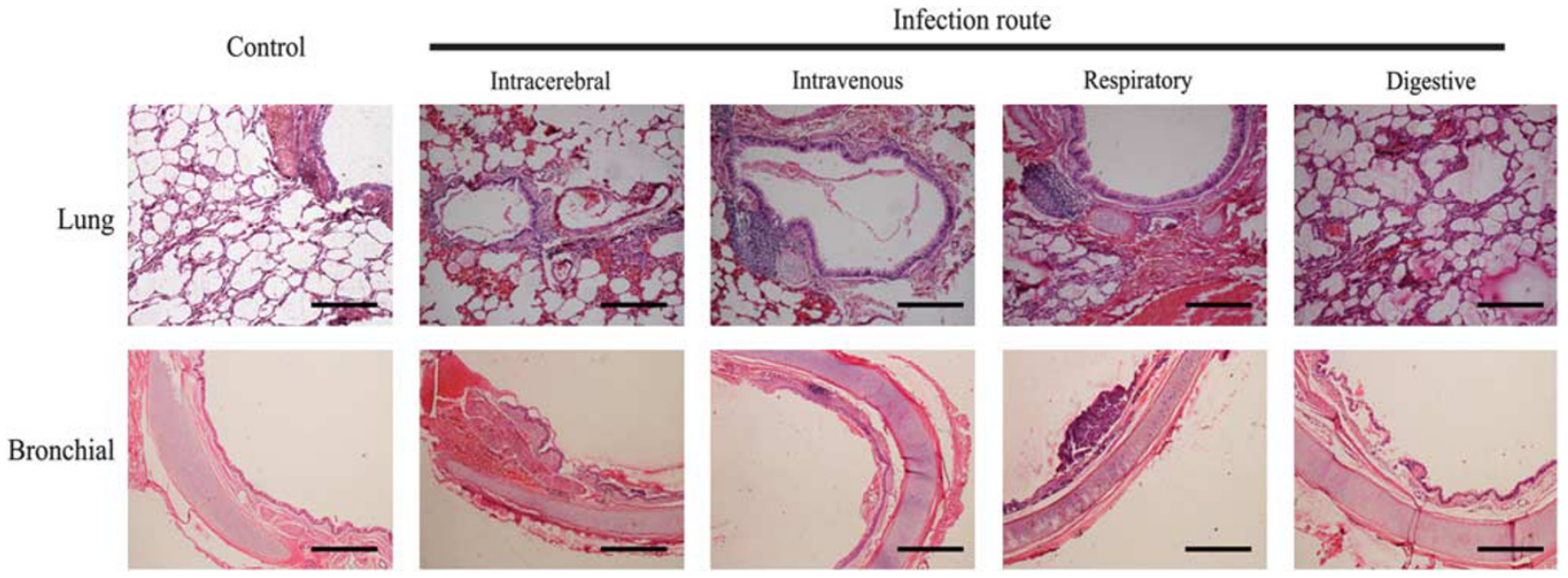

Figure 5 The inflammation-associated pathological observations in respiratory apparatus of EV71-infected rhesus monkeys through intracerebral, intravenous, respiratory and digestive routes. Samples of lung tissue and bronchial tubes were collected on day 10 post infection. The histopathological examinations were performed on tissue sections from organs stained with H\&E according to the protocol. Magnification $\times 100$. Bar, $60 \mu \mathrm{m}$. 
Similarly, inflammation that was associated with pathologies such as massive cell aggregations and lung structure damage was frequently found in the lungs of monkeys that were infected via the respiratory and oral routes (Table 3 and Figure 5); this inflammation was more severe in monkeys that were infected via the respiratory route than in those infected via the digestive route (Table 3). Furthermore, significant damage to the bronchial mucosa and large inflammatory cell aggregations were observed in monkeys that were infected via the respiratory route (Table 3 and Figure 5).

No clear histopathological changes were observed in intestinal organs, such as the stomach and bowels, or other organs, such as the pancreas and spleen, where high viral loads were frequently detected (Table 3 ). Slight inflammatory cell aggregation only appeared in the kidneys and livers of a small number of monkeys (Table 3). All of these histopathological changes were frequently observed from day 4 post infection and became the most severe on days $7-10$ post infection, followed by a gradual recovery (Table 3). Interestingly, the isolation of virus from the feces of monkeys that were infected via all four routes between days 3 and 14 post infection suggests that viral replication occurred in the gastric/intestinal tissue even though no pathological changes were observed there (Table 3 and Supplementary Figure 2).

\section{Viral Antigen Expression in Some Organs of EV71-Infected Rhesus Monkeys}

To further investigate the relationship between EV71 replication in tissues and its pathogenesis, expression of the EV71 antigen in the associated organs of infected rhesus monkeys was investigated. There was high expression of the EV71 antigen in the CNS, lungs and bronchial tubes, which are all areas where pathological changes occurred (Figure 6). Weaker antigen signals were observed in intestinal tissues (Figure 6). These results further confirmed our hypothesis that the virus was respiratory tract tropic. The levels of antigen expression were higher in the tissues of monkeys that were infected via the intracerebral, intravenous and respiratory routes than in the tissues of monkeys that were infected via the digestive route (Figure 6). These findings suggest that virus proliferation within tissues promoted an EV71 infection process that may lead to pathological changes resulting from direct host cell damage or that may cause damage because of immune responses targeting viral antigens in the tissues. Additionally, the titer of specific antibody against EV71 in serum that was detected by neutralization test during infection suggested that viral proliferation in monkeys indeed induced an immune response (Supplementary Table 1).

\section{DISCUSSION}

Enteroviruses, such as poliovirus and coxsackieviruses A and $\mathrm{B}$, are able to initially infect the epithelial cells of the intestine and the associated lymph nodes after entry into the digestive tract. ${ }^{31}$ Subsequently, the viruses proliferate in these primary organs and directly enter the bloodstream to establish systemic viremia, which is frequently linked to pathogenesis and the further infection of target tissues, such as the CNS and the myocardium. ${ }^{32,33}$ In particular, some previous data suggest that poliovirus is not only neuro-tropic but also lymph node tropic. ${ }^{31}$ Although many aspects of EV71 pathogenesis are currently unclear, some preliminary human autopsy reports and well-established murine and cynomolgus monkey models have demonstrated that, in some cases, EV71 pathogenesis occurs by causing neurological lesions in the CNS. ${ }^{18,32,34}$ The observation of EV71-induced encephalomyelitis in human autopsies revealed that the CNS is a focal point for pathological lesions, although the data are limited. ${ }^{28}$ Therefore, a detailed investigation of EV71 pathogenesis relies on the development of an animal model; indeed, mice and cynomolgus monkeys have already been used for this purpose. The murine model revealed the manifestation of progressive paralysis, ruffled fur, humped posture and death after infection with a specific virus strain (mouse-adapted virus strain (MAVS)). ${ }^{19}$ The cynomolgus monkey model revealed neurovirulence manifestations using a temperature-sensitive (ts) strain that was also adapted to this host. ${ }^{5}$

In the current study, the pathogenesis of EV71 in rhesus monkeys that were infected via four inoculation routes was described. The virus strain that was used was originally isolated from a human patient. In general, all of the evidence appears to support the conclusion that EV71 is capable of infecting rhesus monkeys, and the results indicate that EV71 causes a variety of distinct pathological lesions in this animal model. For the first time, detailed timing of an EV71 infection and peak viral viremia in rhesus monkeys that were infected via these four routes were described. The peak viremia values in monkeys that were infected via these four routes had similar tendencies; however, there were some differences (Figure 2). For example, the values for the intracerebrally and orally infected monkeys were not significant compared with the values for monkeys that were infected via the intravenous and respiratory routes (Figure 2). The viremia was similar to that observed in a study of

Figure 6 The viral antigen expression in CNS and other organs from rhesus monkeys infected by $10^{6.5}$ lg CCID ${ }_{50}$ EV71 through intracerebral (monkey ID5346), intravenous (monkey ID5335), respiratory (monkey ID5356) and digestive routes (monkey ID5325). Samples of CNS (cerebra, cervical, thoracic spinal cords, lumbar spinal cords) and other organs (lung, bronchial tubes, liver, small intestine) were collected on day 10 post infection. The samples from CNS and other organs were embedded and frozen in liquid nitrogen, fixed in $3.7 \%$ paraformaldehyde, and treated with hydrogen peroxide (2.5\%). The mouse anti-EV71 antibody and horseradish peroxidase (HRP)-conjugated anti-mouse lgG antibody were used for immunohistochemical assay. Magnification $\times 100$. Bar, $60 \mu \mathrm{m}$. 


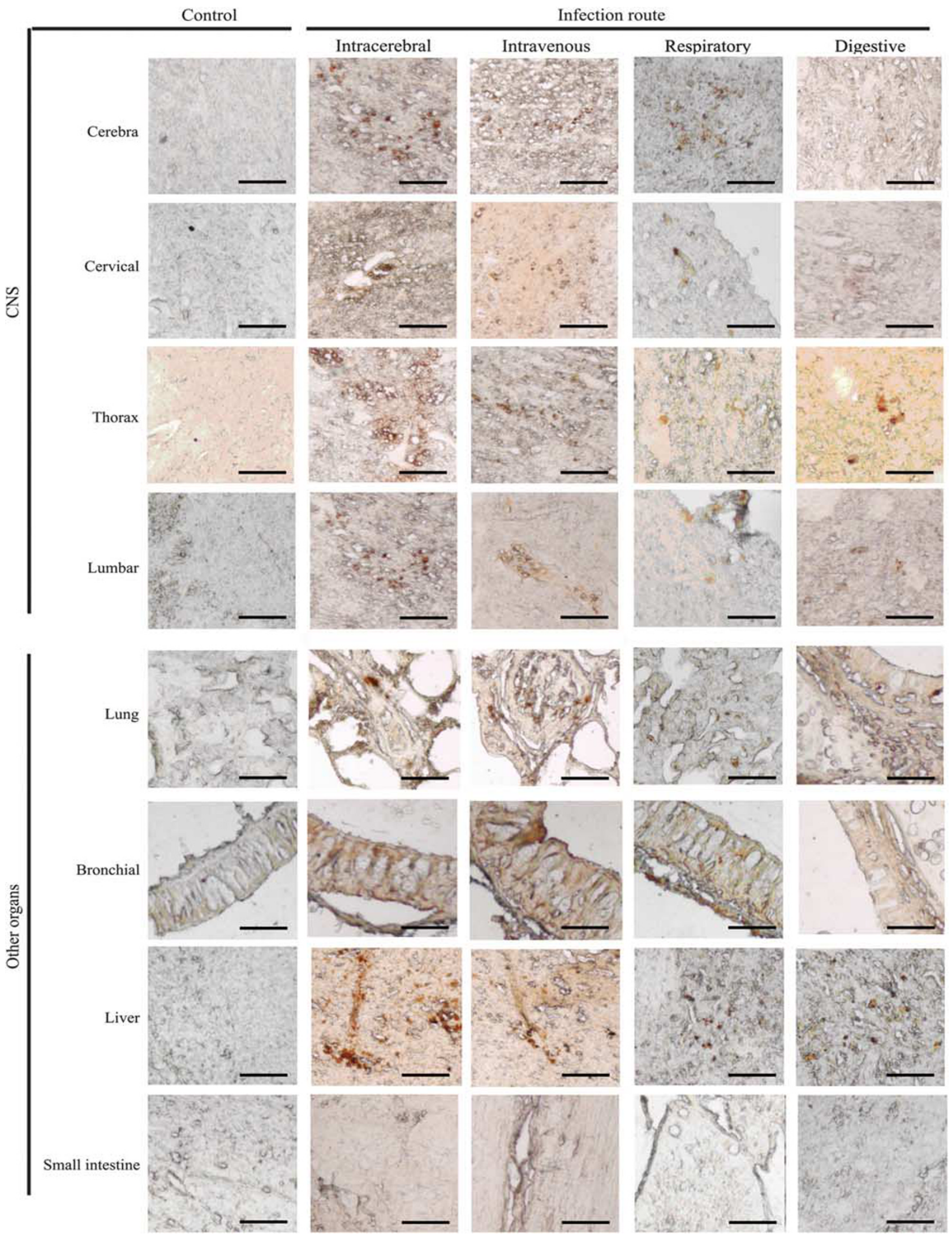


poliomyelitis induced by poliovirus, ${ }^{35}$ which was detected at 1 week post infection and was associated with the presence of virus in throat swabs and feces at the same time that fever, headache and even paralysis were observed. ${ }^{35} \mathrm{~A}$ pathogenic process is implied by the following facts: the viremia on day 4 post infection and its subsequent decrease on days 7 and 8 post infection corresponded to an increase in body temperature in monkeys that were infected via the intracerebral, intravenous and respiratory routes (Figure 1 ); $10^{4-5}$ copies per $100 \mathrm{mg}$ were found in some lymph tissues (such as the axillary lymph node, Figure 3 ); and pathological lesions were observed in the CNS (Figure 4). If a pathogenic process does occur, we hypothesized that the EV71 virus might be transmitted from the primary infection site (probably the bronchial tubes, as shown in this study) to the lymph nodes, resulting in proliferation and dissemination of the virus to target tissues through the blood. In contrast, studies in cynomolgus monkeys and mice revealed that no EV71 replication occurred in the main organs of the animals, except for the CNS. ${ }^{27,29}$ However, it can be argued that oral infection with the strain adapted to mice was capable of inducing virus distribution in some organs, including the heart, lungs and kidneys in mice; ${ }^{32}$ infection with the ts strain via the intravenous route in cynomolgus monkeys ${ }^{27}$ and infection via the intracerebral, intramuscular, intraperitoneal and subcutaneous routes in mice did not yield a similar distribution of the virus. ${ }^{29}$ Our results indicate that virus was present in some organs of the monkeys that were infected via the intravenous and intratracheal routes.

EV71 infection can occasionally lead to neurological sequelae in a small number of patients, ${ }^{36}$ and autopsy data indicate that such neurological complications include brainstem encephalitis, aseptic meningitis and myelitis. ${ }^{32}$ Studies in mice and cynomolgus monkeys have demonstrated that adapted and mutated EV71 strains are capable of inducing neurological lesions and manifesting clinical symptoms in these two animals. ${ }^{5,19}$ The common neuropathological changes that were observed in these studies have provided data to understand the pathogenesis of EV71 encephalomyelitis, but the exact roles of these changes in pathogenesis still need to be investigated. Because the CNS is the main target tissue of this virus, what cells or areas that are attacked by the virus would induce the characteristic disease manifestations in a patient? In humans, severely ill patients usually die of pulmonary edema associated with encephalomyelitis. In the reported mouse and cynomolgus monkey studies, the animals died of paralysis and encephalomyelitis without lesions in the lung tissue. ${ }^{19,27}$ This result is consistent with the fact that damaged neurons and inflammation were concentrated in the motor area. ${ }^{28,29}$ However, damaged neurons and inflammation were also found in motor areas of human autopsy cases and our infected rhesus monkeys. The reason for this result is unclear.

In the present study of EV71 infection in rhesus monkeys, the histopathological lesions and expression of the viral antigen in the CNS indicated the presence of distinct pathological lesions of the neurons and inflammatory cell aggregation in the medulla oblongata, pons, hypothalamus and whole spinal cord of the CNS after the establishment of viremia (Table 2, Figure 6 and Supplementary Figure 1). The characterization of these lesions, typically in monkeys that were infected via the intracerebral route, showed that the neuron retrogression and inflammatory infiltration were focused in the brainstem and the hypothalamus (Figure 4a) and appeared to be related to pulmonary edema (Figure 5). ${ }^{30}$ These pathological changes, although of different severities, were observed in all of the monkeys that were inoculated intravenously and intracerebrally (Table 2 and Figure 6), whereas these changes were only observed in $50 \%$ of the monkeys that were infected via the respiratory route and in $25 \%$ of the monkeys that were infected orally (Table 2). Meanwhile, our work further describes the kinetics of neuropathological progression in rhesus monkeys that were infected with an EV71 strain that originated from a human patient, and these kinetic data provided detailed pathological information on the effects on the CNS in this animal model.

The lesions that were observed in the spinal cord (Figure 4c) were similar to those found in the murine and cynomolgus monkey models. These damaged neurons and the inflammation that was distributed mainly in the anterior horn of the spinal cord suggested that the motor function of infected monkeys could be impaired; such impairment was observed in mice and cynomolgus monkeys but not in our rhesus monkeys. Although we cannot explain this discrepancy, our immunohistopathological examination results were similar to those from human autopsy cases. Permanent paralysis has only rarely been observed in humans. ${ }^{37}$ Based upon the evidence, we conclude that EV71 infection in rhesus monkeys could promote pathological lesions in the CNS, which can be clearly determined according to a pathological index, although no specific clinical symptoms manifested.

However, there were differences among the pathologies of the four groups. The neurological lesions in the intracerebrally infected monkeys appeared to be more severe than the lesions in the other groups and were associated with pulmonary edema in 50\% of intracerebrally infected monkeys (Table 3). Interestingly, intravenous inoculation with EV71 induced the same neurological lesions, but these lesions were associated with the local inflammatory pathological changes in the lung tissue of all of the animals (Table 3). The neurological lesions in monkeys that were infected via the respiratory route were more severe than those in monkeys that were infected orally. Unlike the neurological lesions in the CNS, pathological changes in other organs, except for the lungs and bronchial tubes, were rarely observed. No clear pathological changes were observed in intestinal tissues (Table 3), and the reasons for this result remain unclear. However, pathological changes, including edema, damage to tissue structure and cell infiltration in the lungs, were obvious in all four groups (Table 3). EV71 antigen expression 
in lung tissues, in which viral proliferation was apparently initiated, was observed via immunohistopathological assessment of the tissues (Figure 6). Notably, similar pathological lesions were detected in the bronchial tubes of some infected monkeys (Figure 5 and Table 3). The pathological examination and organ viral load data suggest that EV71 is respiratory tract tropic in rhesus monkeys. These data are seemly different than observations from the murine and cynomolgus monkey models, in which no obvious pathological lesions were found in non-CNS organs. ${ }^{27,29}$ The reason for these differences is unclear. A human autopsy report indicated that the patient had pulmonary edema and inflammation in the lungs. ${ }^{15}$ However, based on the observation of pathological lesions in the respiratory tract of monkeys that were infected via the intravenous, intratracheal and oral routes, it is reasonable to speculate a possible tropism of the virus for the respiratory tract. This hypothesis is supported by the facts that the obvious viremia was induced by viral infection via the intratracheal route and that those lesions were found only in monkeys that were infected via the intratracheal route. However, this speculation requires further investigation and more data for confirmation.

In this study, we demonstrated successful strategies for studying EV71 infection in rhesus monkeys by administration of virus that were isolated directly from a human patient via four different routes. The results led us to conclude that rhesus monkeys may become an important model for studying EV71 infection, which would allow for the direct study of basic pathogenesis and the observation of neurological lesions in the CNS and pathological changes in associated organs. In addition, the fact that EV71 established systemic viremia raised the question of how the subsequent inflammatory responses and pathological changes in the CNS, lung tissues and main target organs could have occurred following viremia. Furthermore, no obvious clinical effects, except for elevated body temperature, were observed, whereas the remarkable viremia and varied profiles of histopathological lesions were confirmed. The reason for this result requires further investigation.

Supplementary Information accompanies the paper on the Laboratory Investigation website (http://www.laboratoryinvestigation.org)

\section{ACKNOWLEDGEMENTS}

This work was supported by National S\&T Major Project (2008ZX10004-014), Grant of Basical Research of CASMS (2010IPB109) and National Basic Research Program (2011CB504903).

\section{DISCLOSURE/CONFLICT OF INTEREST}

The authors declare no conflict of interest.

1. Huang CC, Liu CC, Chang YC, et al. Neurologic complications in children with enterovirus 71 infection. N Engl J Med 1999;341:936-942.

2. Sim AC, Luhur A, Tan TM, et al. RNA interference against enterovirus 71 infection. Virology 2005;341:72-79.

3. Ooi $M H$, Solomon $T$, Podin $Y$, et al. Evaluation of different clinical sample types in diagnosis of human enterovirus 71-associated hand-foot-and-mouth disease. J Clin Microbiol 2007;45:1858-1866.
4. Liu CC, Tseng HW, Wang SM, et al. An outbreak of enterovirus 71 infection in Taiwan, 1998: epidemiologic and clinical manifestations. J Clin Virol 2000;17:23-30.

5. Arita $M$, Shimizu $H$, Nagata $N$, et al. Temperature-sensitive mutants of enterovirus 71 show attenuation in cynomolgus monkeys. J Gen Virol 2005;86(Part 5):1391-1401.

6. Chan LG, Parashar UD, Lye MS, et al. Deaths of children during an outbreak of hand, foot, and mouth disease in Sarawak, Malaysia: clinical and pathological characteristics of the disease. For the Outbreak Study Group. Clin Infect Dis 2000;31:678-683.

7. Shimizu H, Utama A, Yoshii K, et al. Enterovirus 71 from fatal and nonfatal cases of hand, foot and mouth disease epidemics in Malaysia, Japan and Taiwan in 1997-1998. Jpn J Infect Dis 1999;52:12-15.

8. CDC. Deaths among children during an outbreak of hand, foot, and mouth disease-Taiwan, Republic of China, April-July 1998. MMWR Morb Mortal Wkly Rep 1998;47:629-632.

9. McMinn $\mathrm{P}$, Lindsay $K$, Perera $\mathrm{D}$, et al. Phylogenetic analysis of enterovirus 71 strains isolated during linked epidemics in Malaysia, Singapore, and Western Australia. J Virol 2001;75:7732-7738.

10. Huang SC, Hsu YW, Wang $\mathrm{HC}$, et al. Appearance of intratypic recombination of enterovirus 71 in Taiwan from 2002 to 2005. Virus Res 2008;131:250-259.

11. McMinn P, Stratov I, Nagarajan $L$, et al. Neurological manifestations of enterovirus 71 infection in children during an outbreak of hand, foot, and mouth disease in Western Australia. Clin Infect Dis 2001;32: 236-242.

12. Ho M, Chen ER, Hsu KH, et al. An epidemic of enterovirus 71 infection in Taiwan. Taiwan Enterovirus Epidemic Working Group. N Engl J Med 1999;341:929-935

13. Ma S-H, Wang J-J, Shi H-J, et al. Genetic analysis of the VP1 region of human enterovirus 71 strains isolated in Fuyang, China, during 2008. Virol Sin 2009;24:162-170.

14. Li Z-Q, Xu G-C, Han M-F, et al. Clinical analysis of 23 cases of severe hand, foot and mouth disease with neurogenic pulmonary edema in Fuyang City. J Appl Clin Pediatr 2009;24:1403-1408.

15. Lin TY, Hsia SH, Huang YC, et al. Proinflammatory cytokine reactions in enterovirus 71 infections of the central nervous system. Clin Infect Dis 2003;36:269-274.

16. Dolin R. Enterovirus 71-emerging infections and emerging questions. N Engl J Med 1999;341:984-985.

17. Mizuta K, Abiko C, Murata T, et al. Frequent importation of enterovirus 71 from surrounding countries into the local community of Yamagata, Japan, between 1998 and 2003. J Clin Microbiol 2005;43:6171-6175.

18. Nagata N, Shimizu H, Ami $\mathrm{Y}$, et al. Pyramidal and extrapyramidal involvement in experimental infection of cynomolgus monkeys with enterovirus 71. J Med Virol 2002;67:207-216.

19. Wang YF, Chou CT, Lei HY, et al. A mouse-adapted enterovirus 71 strain causes neurological disease in mice after oral infection. J Virol 2004;78:7916-7924.

20. Gorska P. Principles in laboratory animal research for experimental purposes. Med Sci Monit 2000;6:171-180.

21. WHO. Requirements for yellow fever vaccine (Requirements for Biological Substances no. 3, revised 1995). WHO Tech Rep Ser 1998;872:31-68.

22. Arita M, Ami Y, Wakita T, et al. Cooperative effect of the attenuation determinants derived from poliovirus sabin 1 strain is essential for attenuation of enterovirus 71 in the NOD/SCID mouse infection model. J Virol 2008;82:1787-1797.

23. WHO. Procedure for Using the Lyophilized LBM Pools for Typing Enterovirus. Geneva, 1988.

24. Margolis G, Pickett JP. New applications of the Luxol fast blue myelin stain. Lab Invest 1956;5:459-474.

25. WHO. Requirements for poliomyelitis vaccine (oral). WHO Tech Rep Ser 1990;800:30-65.

26. Kao SJ, Yang FL, Hsu YH, et al. Mechanism of fulminant pulmonary edema caused by enterovirus 71. Clin Infect Dis 2004;38:1784-1788.

27. Nagata N, Iwasaki T, Ami $\mathrm{Y}$, et al. Differential localization of neurons susceptible to enterovirus 71 and poliovirus type 1 in the central nervous system of cynomolgus monkeys after intravenous inoculation. J Gen Virol 2004;85(Part 10):2981-2989.

28. Wong KT, Munisamy B, Ong KC, et al. The distribution of inflammation and virus in human enterovirus 71 encephalomyelitis suggests 
possible viral spread by neural pathways. J Neuropathol Exp Neurol 2008;67:162-169.

29. Ong KC, Badmanathan M, Devi $S$, et al. Pathologic characterization of a murine model of human enterovirus 71 encephalomyelitis. J Neuropathol Exp Neurol 2008;67:532-542.

30. Yan JJ, Wang JR, Liu CC, et al. An outbreak of enterovirus 71 infection in Taiwan 1998: a comprehensive pathological, virological, and molecular study on a case of fulminant encephalitis. J Clin Virol 2000;17:13-22.

31. Ouzilou L, Caliot E, Pelletier I, et al. Poliovirus transcytosis through M-like cells. J Gen Virol 2002;83(Part 9):2177-2182.

32. Chen YC, Yu CK, Wang YF, et al. A murine oral enterovirus 71 infection model with central nervous system involvement. J Gen Virol 2004;85(Part 1):69-77.
33. Ren $R$, Racaniello VR. Poliovirus spreads from muscle to the central nervous system by neural pathways. J Infect Dis 1992;166: 747-752.

34. Wang SM, Liu CC. Enterovirus 71: epidemiology, pathogenesis and management. Expert Rev Anti Infect Ther 2009;7:735-742.

35. Horstmann DM, Mc CR, Mascola AD. Viremia in human poliomyelitis. J Exp Med 1954;99:355-369.

36. Alexander Jr JP, Baden L, Pallansch MA, et al. Enterovirus 71 infections and neurologic disease-United States, 1977-1991. J Infect Dis 1994;169:905-908.

37. McMinn PC. An overview of the evolution of enterovirus 71 and its clinical and public health significance. FEMS Microbiol Rev 2002;26: 91-107. 